\title{
Resolvendo o problema multiobjetivo de despacho econômico/ambiental através dos métodos da soma ponderada e de pontos interiores
}

\author{
Elis Goncalves $^{1} \quad$ Diego N. da Silva ${ }^{1} \quad$ Antonio R. Balbo $^{2}$ \\ ${ }^{1}$ Programa de Pós-Graduação em Engenharia Elétrica, Faculdade de Engenharia de Bauru - FEB/UNESP, Bauru, SP \\ E-mail: elisgon01@hotmail.com, diegoitapeva996@hotmail.com \\ ${ }^{2}$ Depto de Matemática, Faculdade de Ciências, FC/UNESP, Bauru, SP \\ E-mail: arbalbo@fc.unesp.br
}

\begin{abstract}
RESUMO
O método previsor corretor primal-dual de pontos interiores, proposto em [4], foi desenvolvido em [6] para Problemas de Programação Não Linear (PPNL) utilizando a estratégia de pontos exteriores relacionada à função barreira logarítmica modificada. Estes métodos têm se mostrado eficientes para a resolução de PPNL's e neste trabalho são aplicados à resolução do problema multiobjetivo de despacho econômico (com ponto de válvula) e ambiental.

O PMDEA é formulado como um problema de otimização que envolve a minimização de dois objetivos conflitantes: custo total de geração $\left(F_{e}\right)$ e custo total de emissão $\left(F_{a}\right)$. O objetivo é otimizar o processo de alocação de energia elétrica entre as unidades geradoras satisfazendo as restrições operacionais e minimizando o custo de geração, além de minimizar a emissão de poluentes na natureza.

A determinação da solução ótima desse tipo de problema é definida na literatura como utópica, [5]. Assim, são necessárias estratégias pré-definidas para a investigação de soluções, das quais destacam-se: o método da soma ponderada (modelo I) e do $\varepsilon_{\text {-restrito (modelo II), que }}$ transformam o problema multiobjetivo em um conjunto de subproblemas mono-objetivos e as soluções ótimas desses subproblemas permitem a determinação de um conjunto de soluções denominadas de eficientes ou não-dominadas para o PMDEA (Pareto-ótimo).

O modelo I utiliza o método da soma ponderada, que considera uma soma balanceada das funções a serem otimizadas e é o método mais simples e mais utilizado na otimização de problemas multiobjetivo. Nele, cada objetivo é multiplicado por um peso e então todos são somados em uma única função objetivo. O PMDEA considera a minimização concomitante das funções $F_{e}$ e $F_{a}$ e é formulado a seguir já considerando a estratégia-método da soma ponderada, de acordo com o modelo I. O modelo II, associado à estratégia de solução denominada de

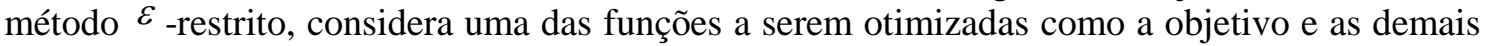
são inseridas como restrições do problema. Esse modelo, que não será apresentado, é objeto de investigação futura.

Neste trabalho a investigação de soluções do PMDEA é feita explorando-se o modelo I, o qual é resolvido através do Método Previsor Corretor Primal Dual de Pontos Interiores com estratégia de Barreira Logarítmica Modificada (MPCPDPIBLM), desenvolvido em [6]. Além do método em destaque, para a resolução do modelo I utilizou-se também um método de aproximante de funções definido em [2], baseado em funções de ativação para suavizar a função de despacho econômico, a qual é não-diferenciável e não-convexa devido à inserção de pontos de válvula e possibilitar a aplicação do MPCPDPIBLM à resolução do modelo I. Os resultados da aplicação feita do método em destaque em um PMDEA contendo 3 geradores são vistos a seguir.
\end{abstract}

Modelo I: PMDEA formulado com a estratégia da soma ponderada 


$$
\begin{gathered}
\quad \begin{array}{c}
\alpha F_{e}+(1-\alpha) F_{a} \\
\text { Minimizar } \\
\text { Sujeito a: } \\
\sum_{i=1}^{n} P_{i}=P_{d} \\
P_{i}^{\min } \leq P_{i} \leq P_{i}^{\max } \\
\text { Em que: } \quad F_{e}=\sum_{i=1}^{n} F_{e P V i}=\sum_{i=1}^{n} a_{i} P_{i}^{2}+b_{i} P_{i}+c_{i}+\sum_{i=1}^{n}\left|e_{i} \operatorname{sen}\left(f_{i}\left(P_{i}^{\min }-P_{i}\right)\right)\right| \\
\quad \text { é a função despacho econômica; } \\
F_{a}=\sum_{i=1}^{n} F_{a_{i}}=\sum_{i=1}^{n} A_{i} P_{i}^{2}+B_{i} P_{i}+C_{i} \text { é a função despacho ambiental } \\
\alpha \in[0,1] \text { é o parâmetro (peso) utilizado pelo método da soma ponderada. }
\end{array}
\end{gathered}
$$

O algoritmo do MPCPDPIBLM foi implementado computacionalmente utilizando o software MATLAB e aplicado a um modelo de 3 geradores, encontrado em [7] e [8].

Utilizando o modelo I solucionado para valores de $\alpha \in[0,1]$ pelo método, é possível perceber que a fronteira de Pareto não está completa, os valores encontrados se concentram próximos aos pontos em que a emissão de poluentes é mínima, em $\alpha=1$ ou o custo é mínimo, em $\alpha=0$.

Assim, a grande dificuldade desta estratégia se dá na escolha dos valores do parâmetro $\alpha$. Isso ocorre, pois a função objetivo definida pela soma ponderada é uma função nãoconvexa, ainda que suavizada nos pontos de válvula. Em [1] é afirmado que, se a função é nãoconvexa, a curva de Pareto sempre terá esse comportamento quando utilizado o método da soma ponderada. É afirmado também que, a estratégia baseada no método $\varepsilon$-restrito para problemas multiobjetivo, é considerada mais adequada e pode fornecer a curva de Pareto completa, sendo esse o próximo passo a ser estudado no trabalho. Uma nova proposta de resolução é apresentada para problemas deste tipo em que uma das funções objetivo é não convexa em [3] e também será explorada no futuro

Palavras-chave: Método Primal- Dual de Pontos Interiores, Método da Soma Ponderada, Despacho Econômico e Ambiental.

\section{REFERÊNCIAS}

[1] Ávila, M. A.; Pfeilsticker, P. C. Problema de Otimização Multiobjetivo.

[2] Chen, C.; Magasarian, O. L. A Class of Smoothing Functions for Nonlinear and Mixed Complementarity Problems, Computational Optimization and Applications, 1996.

[3] Jubril, A. M. Solving Multi-Objective Economic Dispatch Problem Via Semidefinite Programming, IEEE Transactions on power systems, vol. 28, n³, Agosto de 2013.

[4] Mehrotra, S.; Sun, J. An algorithm for convex quadratic programming that requires 0 (n3.5 L) arithmetic operations, Mathematics of Operations Research 15, 342-363, 1990.

[5] Miettinen, K. Nonlinear Multiobjective Optimization. Boston: Kluwer, 1999.

[6] Pinheiro, R. B. N. Um método previsor corretor primal-dual de pontos interiores barreira logaritmica modificada, com estratégias de convergência global e de ajuste cúbico, para problemas de programação não-linear e não-convexa. Dissertação (mestrado) - Faculdade de Engenharia de Bauru, Universidade Estadual Paulista, Bauru, 2012.

[7] Samed,M.M.A. Um Algoritmo Genético Hibrido Co-Evolutivo para Resolver Problemas de Despacho. Tese de Doutorado, UEM, Depto. de Engenharia Química, Agosto de 2004.

[8] Senthil, K.; Manikandan, K. Economic Thermal Power Dispatch with Emission Constraint and Valve Point Effect loading using Improved Tabu Search Algorithm, International Journal of Computer Applications, vol. 3, nº 9, Julho de 2010. 